\title{
Cytological Studies on Calli, in vitro and in vivo Plants of Trachyspermum roxburghianum (DC.) Craib.
}

\author{
Kundamveettil Sreeranjini and John Ernest Thoppil* \\ Genetics and Plant breeding Division, Department of Botany, University of Calicut, \\ Pin Code-673 635, Kerala, India
}

Received January 7, 2005; accepted March 25, 2005

\begin{abstract}
Summary The ploidy levels of the in vitro and the in vivo plants of T. roxburghianum were invariably tetraploid $(2 n=4 x=44)$. The chromosome number of calli were $2 n=2 x=22$ and $2 n=8 x=88$, amidst the normal chromosome number of $2 n=4 x=44$. Changes in the chromosome length, disparity index, variation coefficient and total forma percentage were noticed. The diakinesis and metaphase stages of the in vitro flower showed quadrivalents among bivalents, whereas the same stages of the in vivo plant showed bivalents only.
\end{abstract}

Key words Trachyspermum roxburghianum, Apiaceae, Mitosis, Meiosis, Disparity index, Variation coefficient, Total forma percentage.

Trachyspermum roxburgianum (DC.) Craib., a medicinal plant of the family Apiaceae is used as a culinary herb. The plant is used as a food additive for flavouring, such as in folklore medicine, weight reduction drugs and in artherosclerosis, arthritis, as a cardiotonic, stimulant, carminative and against dyspepsia. The present paper will describe a comparison of karyomorphology and meiotic behaviour of in vivo and in vitro plants and cytological studies on the calli of T. roxburghianum (DC.) Craib.

\section{Materials and methods}

For mitotic studies, young root tips were collected from plants grown in pots at the period between 9-10 a.m. in morning showing peak mitotic activity. The root tips were thoroughly washed in distilled water and pretreated in cytostatic chemicals. Saturated solution of paradichlorobenzene with a trace of aesculin was used for pretreatment. Small quantity of saponin was also added to remove oil particles from the cells. The pretreatment solution is initially chilled to $0-5^{\circ} \mathrm{C}$ for $4-5 \mathrm{~min}$ and the root tips were treated at $12-15^{\circ} \mathrm{C}$ for $2.5-3 \mathrm{~h}$. Then the treated root tips were washed thoroughly with distilled water and fixed in $1: 3$ acetic acid-ethanol mixture overnight. The fixed root tips were washed in distilled water followed by treatment in $1 \mathrm{~N} \mathrm{HCl}$ for $7 \mathrm{~min}$ at room temperature. The root tips were again thoroughly washed to remove last traces of acid. Then root tips were stained in $2 \%$ aceto orcein for $3-4 \mathrm{~h}$ at room temperature. Stained root tips were washed in $45 \%$ acetic acid to destain and then squashed in $45 \%$ acetic acid. The microphotographs of well spread mitotic plates were taken using LEICA-GALEN III Research Microscope attached with a PENTAX photosystem. In in vitro plants and the calli, the same procedure was followed except that treatment in the calli with $1 \mathrm{~N} \mathrm{HCl}$ was for 10 min at room temperature.

To conduct meiotic studies, inflorescence of both in vivo and in vitro plants were collected. Floral buds of appropriate size were removed from the inflorescence and fixed in $1: 3: 6$ (Acetic acid : chloroform : ethyl alcohol) fixative for $1.5 \mathrm{~h}$ and stored in $70 \%$ ethyl alcohol. The anthers of

* Corresponding author, e-mail: ranjini12000@rediffmail.com 
flower buds were removed individually and dissected. They were cut transversely and the content of pollen sacs were extracted on a drop of $2 \%$ acetocarmine by pressing the anther halves. After placing the cover slip, the slide was warmed over an alcohol lamp to improve staining contrast between chromosomes and cytoplasm. Microphotographs of suitable stages were taken.

The karyograms were generated using computer-based program such as Adobe Photoshop. Karyomophometric studies were conducted utilizing Auto CAD and Microsoft Excel. The homologous chromosomes were identified and classified according to Abraham and Prasad (1983). Karyotype formula was deducted from chromosome length, centromere position and length of chromosome with secondary constriction. Disparity index of the chromosomes were calculated after Mohanty et al. (1991) by using the formula

$$
\mathrm{DI}=\frac{\text { Longest chromosome }- \text { Shortest chromosome }}{\text { Longest chromosome }+ \text { Shortest chromosome }} \times 100
$$

The variation coefficient (VC) among the chromosome complements was determined after Verma (1980) as follows:

$$
\mathrm{VC}=\frac{\text { Standard Deviation }}{\text { Mean length of chromosome }} \times 100
$$

The total forma percentage (TF\%) or mean centromeric index value was calculated after Huziwara (1963) by the formula:

$$
\mathrm{TF} \%=\frac{\text { Total sum of short arm length }}{\text { Total sum of chromosome length }} \times 100
$$

\section{Results and discussion}

The karyomorphological changes in the chromosome complement of in vivo, in vitro plants and the calli included changes in the chromosome length, variation coefficient, disparity index and total forma percentage. The ploidy levels of both the in vivo and the in vitro plant were invariably tetraploid $(2 n=4 x=44)$. The chromosome number of the calli observed were $2 n=2 x=22$ and $2 n=8 x=88$ amidst the normal chromosome number i.e., $2 n=4 x=44$ (Figs. $1-5$ ). The cells containing the tetraploid number was more when compared to the other two types of cells observed in the calli. The number of secondary constrictions also showed variations. The parent plant, tetraploid callus and the variant plant showed 4 chromosomes with secondary constriction, whereas the number of chromosomes with secondary constriction was found to be 2 in the diploid callus and 8 in the octaploid callus. Changes in chromosome length, disparity index, variation coefficient and total forma percentage was noticed. The total chromosome length of the parent plant was noticed as $43.0762 \mu \mathrm{m}$, that of the variant was $28.8582 \mu \mathrm{m}$. The total chromosome lengths of diploid, tetraploid and octaploid calli were $16.6340 \mu \mathrm{m}, 28.0124 \mu \mathrm{m}$ and $54.1692 \mu \mathrm{m}$ respectively. The disparity indices observed in the parent and variant were 43.0986 and 30.4527 respectively. The diploid, tetraploid and octaploid calli recorded disparity indices of 29.6822, 38.7633 and 64.9929 respectively. The total forma percentages of the parent, variant, diploid, tetraploid and octaploid calli were estimated as 43.0924, 40.6484, 45.2218, 39.4654 and 45.5318 respectively. The karyotype formulae of the parent plant $\mathrm{A}_{4} \mathrm{C}_{36} \mathrm{D}_{4}$, the variant $\mathrm{A}_{4} \mathrm{C}_{34} \mathrm{D}_{6}$, the diploid callus $\mathrm{A}_{2} \mathrm{C}_{20}$, the tetraploid callus $\mathrm{A}_{4} \mathrm{C}_{28} \mathrm{D}_{12}$ and the octaploid callus $\mathrm{A}_{8} \mathrm{~B}_{2} \mathrm{C}_{68} \mathrm{D}_{10}$ revealed remarkable variations.

The diakinesis of the prophase I, and metaphase I of the in vivo and the in vitro plants were studied (Figs. 6-9). The perfect pairing of homologous chromosomes in the diakinesis stages of 


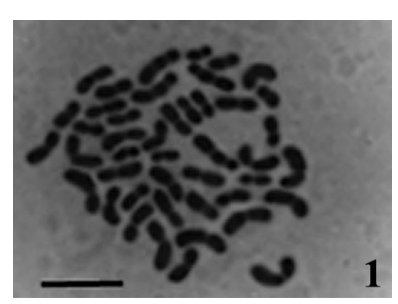

in vivo plant

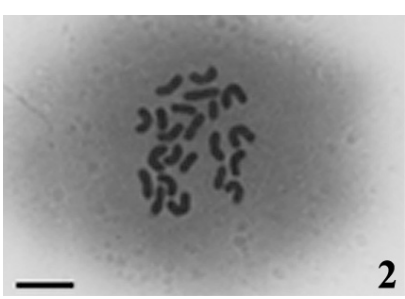

diploid callus

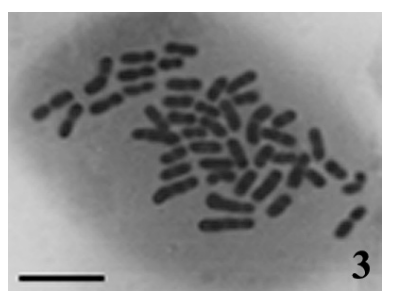

tetraploid callus

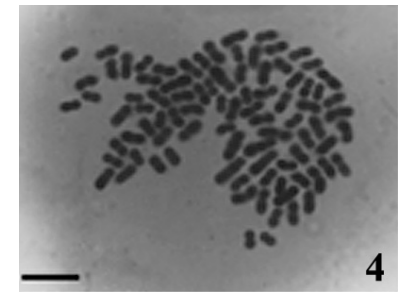

octaploid callus

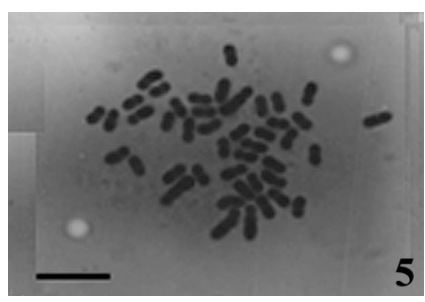

in vitro plant

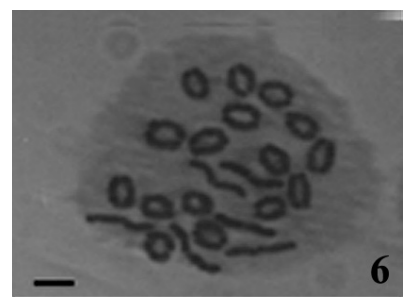

diakinesis of in vivo plant

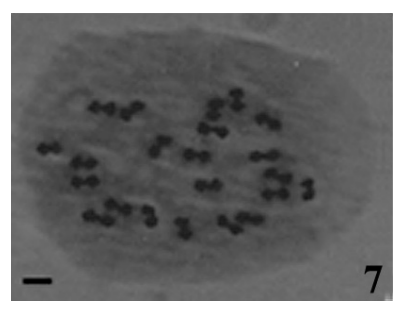

metaphase I of in vivo plant

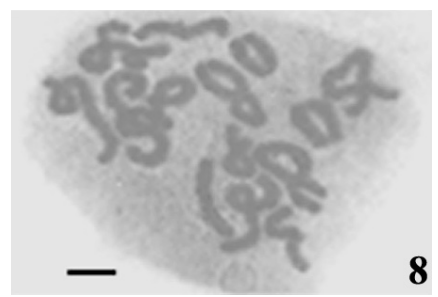

diakinesis of in vitro plant

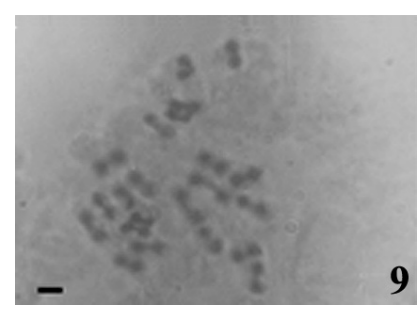

metaphase I of in vitro plant

Figs. 1-9. Mitotic and meiotic stages of in vivo and in vitro plants of Trachyspermum roxburghianum (DC.) Craib. Figs. 1-5: Mitotic metaphase stages, Figs. 6-9: meiotic stages. Bar $=1 \mu \mathrm{m}$.

meiosis in the in vivo plant point towards the very balanced nature of karyotype. The in vivo plant showed no cytological abnormalities in meiotic cells and formed 22 bivalents at metaphase I of PMCs, whereas the in vitro plant showed 18 bivalents and 2 quadrivalents. The diakinesis stage of prophase I of in vivo flower showed 22 normal bivalents, whereas the in vitro flower consisted of 10 bivalents and 6 quadrivalents.

The change in the chromosome length and ploidy level in the calli may be the aftermath of cryptic changes, which may arise due to in vitro stress produced in the altered culture environment. Retrotransposon activation, deletion, duplication and inversions may significantly contribute to the change in the physical size of the genome. Comparatively longer chromosomes in the karyotype seem to be a primitive characteristic feature (Dasgupta and Datta 1976). The higher individual chromosome length in the in vivo plant seems to be in consonance with this, which may suggest considering it as primitive when compared to the other samples. The number of submetacentric chromosomes were found to be higher in the in vitro plant when compared with that of in vivo plant. An increase of submetacentrics at the expense of metacentrics leads to asymmetry of the karyotype, which is an advanced condition (Stebbins 1958).

In the present investigation, meiotic aberrations were observed. Aberrant meiotic associations may be due to some spontaneous multiple translocations which might have arisen due to change in many genes or due to the special genetic constitution or changes in the internal or external environment leading to the abnormal associations in the PMCs. The presence of multivalents in the normal tetraploid species leads to the assumption of structural heterozygosity involving segmental interchanges. The same was reported by Srivastava and Naithani (1964). From the evolutionary stand- 
point, meiotic instability may be considered as an evidence of variation.

\section{References}

Abraham, A. and Prasad, I. 1983 A system of chromosome classification and nomenclature. Cytologia 48: 95-101.

Dasgupta, A. and Datta, P. C. 1976 Cytotaxonomy of Piperaceae. Cytologia 41: 697-706.

Huziwara, Y. 1963 The karyotype analysis in some genera of Compositae X: The chromosomes of some European species of Aster. Bot. Mag. Tokyo 75: 143-150.

Mohanty, B. D., Ghosh, P. D. and Maity, S. 1991 Chromosome analysis in cultured cells of barley (Hordeum vulgare L.). Structural alterations in chromosomes. Cytologia 56: 191-197.

Srivastava, L. M. and Naithani, S. P. 1964 Cytogenetical studies in certain minor pulses and beans. Cytologia 29: $453-464$.

Stebbins, G. L. 1958 Longevity, habitat and release of genetic variability in higher plants. Cold Spring Harb. Symp. Quart. Biol. 23: 365-378.

Verma, B. N. 1980 Karyotype analysis in three species of Rhizoclonium Kurtz. Cytologia 45: 433-440. 